\title{
Truncation Errors in the Spherical Harmonic Analysis of the Geomagnetic Field and the Problem of Downward Extrapolation
}

\author{
P. H. ROBERTS \\ Yerkes Observatory, Williams Bay, Wisconsin, U.S.A.* \\ and \\ S. ScOTT \\ Rutherford College of Technology, Newcastle upon Tyne, England \\ (Received Oct. 1, 1963)
}

\begin{abstract}
The problem of extrapolating the geomagnetic field to the core boundary is considered, and the problem of suppressing the concomitant amplification of errors is examined in some detail. It is proved that if the extrapolation is accomplished by spherical harmonic analysis, the familiar least squares (or $\chi^{2}$-minimization) process of deriving the harmonic coefficients is open to theoretical objections. An alternative method is proposed which evades these criticisms.
\end{abstract}

\section{Introduction}

The importance of determining the structure of the magnetic field deep within the earth has led to several direct attempts to represent the field by physical sources within the core (see, for example, McNish (1940); Lowes and Runcorn (1951)). The exclusion of sources within the mantle (apart from local anomalies) is justified, on theoretical grounds, by the likelihood of its small conductivity and susceptibility and, on observational grounds, by the absence of all but the very long and very short (anomaly) wave-lengths in the Fourier analysis of track-line data (Alldredge and Van Voorhis, 1961). This, in principle, allows the unique extrapolation of the geomagnetic field from the earth's surface to the core boundary. However, the direct method of extrapolation, by dividing the field into its harmonic components and extrapolating these individually, has generally been eschewed because of the amplification factor $(a / b)^{n+1}$ by which the random and anomaly errors in the $n$th harmonic are amplified during the extrapolation from the Earth's surface $(r=a)$ to the core boundary $(r=b)$. However, two points should be made concerning this objection.

First, looked at purely as a mathematical problem, the extrapolation process is one of integrating an elliptic differential equation (i.e. Laplace's equation) under Cauchy type bountary conditions (i.e., in essence, under the specification of the potential and its

* Now at Department of Mathematics, University of Newcastle upon Tyne, England. 
vertical derivative at the earth's surface) towards a region in which sources lie (i.e. the core). This is a difficult problem in numerical analysis about which little is known. It has long been realised that extrapolation by methods of step by step integration must fail since they are unstable no matter how small the steps are made (cf. e.g. Courant, Friedrichs and Lewy (1928)). Moreover, no matter what process of extrapolation is adopted, this very fundamental difficulty will persist in a more or less severe form, and must inevitably lcad to an increase of the probable error in the field determination at the core boundary over its value at the earth's surface. The ideal extrapolation process is one which minimizes this probable error. (This, it will transpire below, will not, in general, minimize the mean square error between the data and their representation at the earth's surface.) There is no reason to suppose that extrapolation by analysis into physical sources in the core should be superior to a harmonic analysis properly carried out; indeed it may be anticipated that the greater preponderence of short wave-lengths at the core boundary produced by the discrete sources will give rise to a larger probable error than that of the harmonic analysis.

Second, even though the errors in the $n$th harmonic are amplified by $(a / b)^{n+1}$ during the extrapolation, the harmonic itself is amplified by the same amount, and the signal/noise ratio is left unchanged. Thus, provided the process of extracting harmonic coefficients does not itself greatly increase the noise (and provided the noise of large harmonic number is cut off (see below), harmonic analysis should provide a reliable basis for extrapolation.

In discussing ways and means of reducing noise in harmonic coefficients, we must recognise immediately that, since the noise spectrum is expected to be white, there will exist a harmonic number $p$ beyond which the spectrum of the observation is dominated by noise. The more accurately and completely the geomagnetic field is measured, and the effects of local anomalies eliminated, the greater $p$ will be, and the smaller the probable error in the extrapolated field will become. Since harmonics dominated by noise are of no interest, it is natural to divide the hypothetical infinite harmonic series into a 'representation series', which includes all harmonics up to, and including, $n=p$, and a 'discard series', which includes all harmonics beyond $p$. For the purpose of extrapolation we seek to determine the coefficients of the representation series as accurately as possible. Thus, we do not adopt least squares or $\chi^{2}$-minimization procedures because these rely on 'telescoping' the discard series into the representation series in order to imitate the observatory data as accurately as possible. Although this 'contamination' is of no significance when such a series is used for data representation, it introduces systematic errors in the harmonic coefficients which are amplified in the process of downward extrapolation.

Quantitative support for these ideas is given below, particularly in Sect 3. Meanwhile, we merely observe in passing that the elimination of harmonic contamination which we advocate is the logical culmination of the recognized practice of modifying observatory data by results of local surveys. This process removes noise of very high harmonic numbers which is set up by magnetic anomalies in the vicinity of the observatory, which, if not eliminated, would be ascribed erroneously to the harmonics of the representation series, and would lead to serious discrepancies even in the representation of the geomagnetic field 
over the earth's surface.

The best method of reducing contamination is not known to us. We do, however, establish an improvement over least squares and $\chi^{2}$-minimization by a process which we will term 'exact fitting and discard'. Although, as we have seen, the harmonic terms beyond $n=p$ have no objective meaning, we will nevertheless make the first $N$ terms of a harmonic series fit exactly the $N$ data points given; all the terms in the series beyond $n=p$ are then discarded. Should it happen that the data points are so happily situated that the $n$ spherical harmonics are orthogonal with respect to them, the results derived are identical to those of a least-square determination of the harmonics $n \leqslant p$. Otherwise this is not the case. It is still true that, no matter where the $N$ data points are located, it is possible to construct $N$ linear combinations of the $N$ harmonics which are orthogonal to the data points (cf. e.g. Kendall (1943), p. 150), and that, in fitting a series of $P$ (say) of these combinations to the data, the least squares method and the method of exact fitting and discard are identical. However, each of these $P$ combinations would, in general, involve all $N$ harmonics and, to translate all the results back to the harmonics themselves, it would be necessary that $P=N$.

The $\chi^{2}$-minimization method properly treats correlations of errors between observatories, and it might be argued that, to achieve a like result, some refinement of the method of exact fitting and discard should be introduced. However, this is not the case; of the two sources of correlation present, one, due to fields of external origin, though world-wide is transitory and averages out when mean values of observatory data are taken, while the other, due to local anomalies, though permanent is on a spatial scale too small compared with observatory spacing to be important. It should be noticed that, although, for simplicity, we consider only the case in which the data points are regularly spaced over the earth's surface, similar arguments apply in the case of irregular spacing. This may be of some practical interest, since direct use of observatory dataf (unmodified except for the removal of local anomalies) eliminates noise set up during interpolation to regularly spaced grid points. However, for the present (theoretical) purpose, the complications added to the discussion by considering irregularly spaced grid points cloud the main issues and seem best avoided. We assume, therefore, that the grid points are at the intersection of equally spaced lines of longitude and symmetrically placed (with respect to the equator) lines of latitude. The fitting process is carried out in two stages: first, analysis around each line of latitude into Fourier series; second, analysis of the dependence of the coefficients in the Fourier series upon latitude. The first process is simple, well-known and introduces negligible contamination; it is nevertheless briefly treated in Sect. 2 so that we may take the opportunity of laying emphasis where, we believe, emphasis should be laid. The principles involved are also of importance in Sect. 3, where the second process of latitude dependence is discussed, and the contamination introduced by two different methods are compared.

$\dagger$ A recent paper, by Fougere (1963), in which the normal, least squares, equations, are solved by an orthogonalising process, describes an analysis of model data distributed over 66 observatories. 


\section{Analysis of longitude dependence}

In its mathematically simplest form, the problem faced is one of representing a (bounded) function $V^{*}(\theta, \phi)$ on the surface of a sphere by a series of the form

$$
V^{*}(\theta, \phi)=\sum_{n=0}^{\infty} \sum_{m=0}^{n}\left(g^{*}{ }_{n, m} \cos m \phi+h^{*}{ }_{n, m} \sin m \phi\right) p_{n}^{m}(\theta)
$$

Here $\theta$ denotes co-latitude measured from the North pole, $\phi$ denotes longitude, $p_{n}^{m}(\theta)$ denotes the associated Legendre polynomial, and $g^{*}{ }_{n, m}$ and $h^{*}{ }_{n, m}$ are constants to be determined. In equation (1) as elsewhere in this paper, we use the Schmidt normalization of the associated Legendre polynomials for which (see, for example, Chapman and Bartels, volume 2, 1940, p. 610)

$$
\int_{0}^{\pi} p_{n}^{m}(\theta) p_{n \prime}^{m}(\theta) \sin \theta d \theta=\frac{2}{(2 n+1)}\left(2-\delta_{m}^{0}\right) \delta_{n}^{n}
$$

(Here $\delta_{b}^{a}$ denotes the Kronecker delta which is zero unless $a$ and $b$ are equal, and is then equal to unity). For later reference we will divide expansion (1) thus:

$$
V^{*}(\theta, \phi)=\sum_{m=0}^{\infty}\left(G^{*}{ }_{m}(\theta) \cos m \phi+H^{*}{ }_{m}(\theta) \sin m \phi\right)
$$

where

$$
G^{*}{ }_{m}(\theta)=\sum_{n=m}^{\infty} g^{*}{ }_{n, m} p_{n}^{m}(\theta), \quad H^{*}{ }_{m}=\sum_{n=m}^{\infty} h^{*}{ }_{n, m} p_{n}^{m}(\theta)
$$

(In applying the analysis to the earth's magnetic field, the $n=0$ term does not enter into (1).)

In principle, the determination of $g^{*}{ }_{n, m}$ and $h^{*}{ }_{n, m}$ is merely a matter of integration:-

$$
\begin{aligned}
g^{*}{ }_{n, m} & =\frac{(2 n+1)}{4}\left(1+\delta_{m}^{0}\right) \int_{0}^{\pi} G_{m}^{*}(\theta) p_{n}^{m}(\theta) \sin \theta d \theta \\
& =\frac{(2 n+1)}{4 \pi} \int_{0}^{2 \pi} d \phi \int_{0}^{\pi} V^{*}(\theta, \phi) p_{n}^{m}(\theta) \cos m \phi \sin \theta d \theta \\
h^{*}{ }_{n, m} & =\frac{(2 n+1)}{4} \int_{0}^{\pi} H^{*}(\theta) p_{n}^{m}(\theta) \sin \theta d \theta \\
& =\frac{(2 n+1)}{4 \pi} \int_{0}^{2 \pi} d \phi \int_{0}^{\pi} V^{*}(\theta, \phi) p_{n}^{m}(\theta) \sin m \phi \sin \theta d \theta
\end{aligned}
$$

These results can also be derived formally by choosing $g^{*}{ }_{n, m}$ and $h^{*}{ }_{n, m}$ so as to minimize

$$
L^{*}=\int_{0}^{2 \pi} d \phi \int_{0}^{\pi}\left[V^{*}(\theta, \phi)-\sum_{n=0}^{\infty} \sum_{m=0}^{n}\left(g^{*}{ }_{n, m} \cos m \phi+h^{*}{ }_{n, m} \sin m \phi\right) p_{n}^{m}(\theta)\right]^{2} \sin \theta d \theta
$$

In practice, we may know $V$ only at a number of grid points. Suppose that these are located in the following way: draw $s$ semi-circles of longitude each separated from its neighbours by an angle of $2 \pi / s$, and draw $2 q+1(q \gg 1)$ circle of latitude, of which poles and equator count as three, the remainder being symmetrically placed with respect to the equator. The grid points are the $N=2 q s-s+2$ points of intersection: their coordinates are $\left(\theta_{\alpha}, 2 \pi \beta / s\right)$ where $\alpha$ runs from 0 to $2 q\left(\theta_{0}=0, \theta_{2 q}=\pi\right)$ and $\beta$ runs from 1 to $s$. To the values $V_{a, \beta}$ we attempt to fit a series of the form 


$$
V(\theta, \phi)=\sum_{n=0}^{n} \sum_{m=0}^{n}\left(g_{n, m} \cos m \phi+h_{n, m} \sin m \phi\right) p_{n}^{m}(\theta)
$$

where $p^{2}<2 s q-s+2$ and $2 p \leqslant s-1$. (In geomagnetic applications, it is customary to set $g_{0,0}=0$.) We may note in passing that Chakrabarty (1954) has objected to this technique, and has preferred to calculate $g^{*}{ }_{n, m}$ and $h^{*}{ }_{n, m}$ by quadrature approximations to the integrals (5) and (6). However it is clear that every form of numerical integration can be equated exactly to a parexic curve fit, from the step function of the mid-ordinate rule to the various polynomial curves, followed by an elementary integration. Thus nothing, either new or different, would result by a consideration of the integration method, and we have found it simpler to present our discussion in terms of fitting truncated series of the form (8) to the data.

We may conveniently divide the process of fitting into two stages. First, fix attention on one $\theta_{\alpha}$ and fit a series

$$
V\left(\theta_{\alpha}, \phi\right)=\sum_{m=0}^{n}\left[G_{m}\left(\theta_{\alpha}\right) \cos m \phi+H_{m}\left(\theta_{\alpha}\right) \sin m \phi\right]
$$

Second, having repeated the process for all $\theta_{\alpha}$, fit $G_{m}\left(\theta_{\alpha}\right)$ and $H_{m}\left(\theta_{\alpha}\right)$ by the series:-

$$
G_{m}(\theta)=\sum_{n=m}^{n} g_{n, m} p_{n}^{m}(\theta), \quad H_{m}(\theta)=\sum_{n=m}^{n} h_{n, m} p_{n}^{m}(\theta)
$$

We conclude this section by a brief discussion of the first of these processes.

The fitting of exact data $V_{\beta}$ at equally spaced $\left(\phi=\phi_{\beta}=2 \pi \beta / s, \beta=1\right.$ to $\left.s\right)$ grid points by a series of the form

$$
V(\phi)=\sum_{m=0}^{n}\left(G_{m} \cos m \phi+H_{m} \sin m \phi\right)
$$

can be carried out in two ways, which we will presently show are essentially the same. The first is a process of exact fitting and discard. Let $t$ be the smallest integer greater than or equal to $(s-1) / 2$. Then a series of the form

$$
V(\phi)=\sum_{m=0}^{t}\left(G_{m} \cos m \phi+H_{m} \sin m \phi\right)
$$

can certainly be made to fit the values of the grid points exactly: there are $s$ grid points and $2 t+1(>s)$ constants $G_{m}$ and $H_{m}$. The series (11) is derived simply from (12) by discarding all harmonics after the $p$ th. The assumption of a finite series (12) has the effect of redistributing the harmonics $G^{*}{ }_{t+1}, G^{*}{ }_{t+2} \ldots \ldots$ among the harmonics $G_{0}{ }^{*}$ to $G_{t}{ }^{*}$ (similarly for $H^{*}$ ). To see this, we first simplify the discussion by assuming $s=2 t+1$ so that the constants $G_{m}$ and $H_{m}$ are uniquely determined from $V\left(\phi_{\beta}\right)$ by the $(2 t+1)$ simultaneous equations

$$
\sum_{m=0}^{t}\left(G_{m} \cos m \phi_{\beta}+H_{m} \sin m \phi_{\beta}\right)=V\left(\phi_{\beta}\right), \quad \beta=1,2 \ldots(2 t+1)
$$

Hence, since $\Sigma_{\beta} \cos m \phi_{\beta} \cos m^{\prime} \phi_{\beta}=s \delta_{m}^{m \prime}\left(1+\delta_{m}^{0}\right) / 2$,

$\Sigma_{\beta} \sin m \phi_{\beta} \sin m^{\prime} \phi_{\beta}=s \delta_{m}^{m \prime}\left(1-\delta_{m}^{0}\right) / 2$,

$\Sigma_{\beta} \sin m \phi_{\beta} \cos m^{\prime} \phi_{\beta}=0$ 
we can solve immediately

$$
G_{m}=\left(2-\delta_{m}^{0}\right) / s \Sigma_{\beta} V\left(\phi_{\beta}\right) \cos m \phi_{\beta}, \quad H_{m}=(2 / s) \sum_{\beta} V\left(\phi_{\beta}\right) \sin m \phi_{\beta}
$$

Now substituting for $V\left(\phi_{\beta}\right)$ by equation (3) and using (14), we find that

$$
\begin{aligned}
& G_{m}=G^{*_{m}}+G^{*_{s-m}}+G^{*_{s+m}}+G^{*_{2 s-m}}+G^{*_{2 s+m}}+\ldots \ldots \\
& H_{m}=H^{*_{m}}-H^{*_{s-m}}+H^{*_{s+m}}-H^{*_{2 s-m}}+H^{*_{2 s+m}} \ldots \ldots
\end{aligned}
$$

(cf. Chapman and Bartels, volume 2, 1940, p. 559). Thus, the coefficients $G_{m}$ and $H_{m}$, which we have calculated, are not equal to the coefficients $G^{*}{ }_{m}$ and $H^{*}{ }_{m}$, which we require; they are 'contaminated' by the higher harmonics $G^{*}{ }_{s \pm m}$ and $H^{*}{ }_{s \pm m}$. However, in the case of the earth's field, where it is reasonably certain that the fields have a deep internal origin, we may expect that the coefficients $G^{*}{ }_{m}$ and $H^{*}{ }_{m}$ decrease rapidly as $m$ increases (local anomalies having been excluded) and we may be sure that, for small $m$, the coefficients we require, namely $G^{*}{ }_{m}$ and $H^{*}{ }_{m}$, are given with little error by the coefficients we have calculated, namely $G_{n}$ and $H_{m}$. Also, the fact that the high harmonics (e.g. $G_{t}$ ) are seriously $\dagger$ influenced by the higher harmonics (e.g. $G^{*}{ }_{s-t}$ ) is of no significance since we have already agreed that terms beyond the cut-off point $p(\ll t)$ are of no significance, and should be discarded. Thus the contamination implied by (16) is indeed small, and it is not likely that a refinement of the present method would reduce it significantly. We may note in passing that our aim in Sect. 3 is to produce an equally good result for the analysis of the latitude dependence.

The second method referred to above is to fit series (11) by 'least squares', i.e. by minimizing

$$
M=\sum_{\beta}\left[V\left(\phi_{\beta}\right)-\sum_{m=0}^{p}\left(G_{m} \cos m \phi_{\beta}+H_{m} \sin m \phi_{\beta}\right)\right]^{2}
$$

The values of $G_{m}$ and $H_{m}$ are given by $\partial M / \partial G_{m}=\partial M / \partial H_{m}=0$, i.e. by

$$
\begin{aligned}
& \sum_{\beta} \cos m \phi_{\beta} \sum_{m=0}^{n}\left(G_{m}, \cos m^{\prime} \phi_{\beta}+H_{m} \sin m^{\prime} \phi_{\beta}\right)=\sum_{\beta} V\left(\phi_{\beta}\right) \sin m \phi_{\beta} \\
& \sum_{\beta} \sin m \phi_{\beta} \sum_{m=0}^{n}\left(G_{m}, \cos m^{\prime} \phi_{\beta}+H_{m}, \sin m^{\prime} \phi_{\beta}\right)=\sum_{\beta} V\left(\phi_{\beta}\right) \sin m \phi_{\beta}
\end{aligned}
$$

Because of the orthogonality (14) of the functions $\cos m \phi_{\beta}$ and $\sin m \phi_{\beta}$ with respect to the grid points, and for no other reason, (18) reduces to (15), and (16) follows.

\section{Analysis of Latitude dependence}

We now discuss the problem of fitting series of the form

$$
G_{m}(\theta)=\sum_{n=m}^{p} g_{n, m} p_{n}^{m}(\theta), \quad H_{m}(\theta)=\sum_{n=m}^{n} h_{n, m} p_{n}^{m}(\theta)
$$

$\dagger$ This gives another reason, apart from noise, why $t$ must be chosen to be much greater than $p$. On these grounds we may criticise Gauss' original analysis. Gauss took $p=4, t=4, s=9$. The coefficients $G_{4}$ and $H_{4}$ were therefore seriously influenced by $G_{5}{ }^{*}$ and $H_{5} *$. There can be no such objection to Vestine's analysis (1947), since, for this, $p=6, t=18$ and $s=36$. 
to the $(2 q+1)$ values of $G_{m}\left(\theta_{\alpha}\right)$ and $H_{m}\left(\theta_{\alpha}\right)$ derived in the analysis of Sect. 2 . When the aim is strictly that of empirical interpolation it is, of course, possible with an arbitrary assignment of grid points $\theta_{\alpha}$, to construct polynomials up to degree $2 q$ which are orthogonal with respect to those grid points (cf. e.g. Kendall, 1943, p. 150). Although such a procedure would facilitate the numerical computations, the polynomials concerned would not be simply related to spherical harmonics and, on translating the results to the forms (10), it would be difficult to make certain that $g_{n, m}$ and $h_{n, m}$ were not seriously 'contaminated' by neighbouring harmonics; indeed, as we will demonstrate presently, such contamination does take place in the least square method. The emphasis, then, is not upon orthogonal polynomials, 'rigid' coefficients and least squares, but upon minimising the contamination of the ascertainable harmonics.

Let us fix attention on $G_{0}(\theta)$. (We will omit the suffix $o$ in what follows). The methods for the remaining $G_{m}$ and $H_{m}$ are exactly similar. First, we consider a method of exact fitting. A series of the form

$$
G(\theta)=\sum_{n=0}^{2 q} g_{n} p_{n}(\theta)
$$

can certainly be made to fit exactly the values at the grid points uniquely: there are $2 q+1$ grid points and $2 q+1$ constants $\theta_{a}$. The series (10) for $G_{0}$ can then be derived simply by omitting all harmonics after the $p$ th. The equations determining $g_{n}$ are simply

$$
\sum_{n=0}^{2 q} p_{n}\left(\theta_{\alpha}\right) g_{n}=G\left(\theta_{\alpha}\right), \quad \alpha=1,2 \ldots(2 q+1),
$$

or, in matrix form,

$$
A g=G,
$$

where $A_{\alpha, r}$ is a $(2 q+1) X(2 q+1)$ square matrix and $g_{r}$ and $G_{\alpha}$ are column vectors of $(2 q+1)$ elements

$$
A_{\alpha, \gamma}=P_{\gamma}\left(\theta_{\alpha}\right), \quad G_{\alpha}=G\left(\theta_{\alpha}\right) .
$$

Denote the inverse of $A$ by $A^{-1}$. According to (21), we have

$$
g=A^{-1} G,
$$

and, on substituting the exact expression for $G$ as given by (4), we find $(n<2 q)$

$$
g_{n}=g_{n}^{*}+\sum_{l=2 \eta+1}^{\infty} g_{l}^{*} \sum_{\alpha} A_{n, \alpha}^{-1} p_{l}\left(\theta_{\alpha}\right)
$$

i.e.

$$
g_{n}=g_{n} *+\sum_{l=2 q+1}^{\infty} \Delta_{n, l} g_{l}^{*},
$$

where $\Delta_{n, l}(n=0$ to $2 q ; l>2 q)$ is the solution of the equations

$$
\sum_{n=0}^{2 q} p_{n}\left(\theta_{\alpha}\right) \Delta_{n, l}=p_{l}\left(\theta_{\alpha}\right)
$$

Thus, as in the analysis of Sect. 2 , we may be sure that, if $p \ll 2 q$, the coefficients we require, namely $g_{n} *(n \leqslant p)$, are given with little error by the coefficients we have calculated, namely 
$g_{n}$. By suitable choice of grid points, the position may even be improved further. For example, if we choose $\theta_{\alpha}$ to be the $2 q+1$ zeros of $p_{2 q+1}(\theta), \Delta_{n, 2 l+1}$ vanishes for all $n$, and the first harmonic to adversely affect $g_{n}$ becomes $g^{*}{ }_{2 q+3}$ instead of $g^{*}{ }_{2 q+1}$. This particular method of data fitting is equivalent to using Gauss' quadrature method to integrate over $\theta$ in (5) and (6). It suffers from the practical disadvantage that, for each value of $m$, the analysis of the longitude dependence must be carried out afresh, since the data circles $\theta$ must be chosen differently (by $p_{2 q+1}^{m}(\theta)=0$.)

We consider next the least square method. This involves minimising

$$
N=\sum_{\alpha} W\left(\theta_{\alpha}\right)\left[G\left(\theta_{\alpha}\right)-\sum_{n=0}^{n} g_{n} p_{n}\left(\theta_{\alpha}\right)\right]^{2}
$$

where $W\left(\theta_{\alpha}\right)$ is a weight factor. If the data at the grid points are considered to be equally reliable, the best choice of $W\left(\theta_{\alpha}\right)$ is the scale factor $\sin \theta_{\alpha}$ of the coordinate system, for then, in the limit of equally spaced grid points and $q \rightarrow \infty$, (27) reduces to (5), and $g_{n, m} \rightarrow g^{*}{ }_{n, m}$ and $h_{n, m} \rightarrow h^{*}{ }_{n, m}$ (see (35) below). It might be argued that, in geomagnetic practice, the best choice of $W\left(\theta_{\alpha}\right)$ would be proportional to the number of observatories near (in some sense) the line of latitude $\theta_{\alpha}$ (Dyson and Furner, 1923). A weighting factor should then, of course, be also introduced into the longitude analysis of Sect. 2 (see Chakrabarty, 1954) and the results of Sect. 2 appropriately modified. However, Chakrabarty (1954) has commented on the fact that two determinations of the coefficients, namely those by Vestine (1947) and Spencer Jones and Melotte (1953), were nearly the same despite the different weightings employed. It is therefore conceivable that the precise choice of $W\left(\theta_{\alpha}\right)$ is not critical. The values of $g_{n}$ are given by $\partial N / \partial g_{n}=0$, i.e. by

$$
\sum_{\alpha} W\left(\theta_{\alpha}\right) p_{n}\left(\theta_{\alpha}\right)\left[\sum_{n \prime=0}^{p} g_{n \prime} p_{n \prime}\left(\theta_{\alpha}\right)\right]=\sum_{\alpha} W\left(\theta_{\alpha}\right) p_{n}\left(\theta_{\alpha}\right) G\left(\theta_{\alpha}\right), \quad(n=0,1 \ldots p)
$$

This may be rewritten

$$
\sum_{n=0}^{n} B_{n, n} g_{n^{\prime}}=C_{n}, \quad(n=0,1 \ldots p)
$$

or in matrix form

$$
B g=C,
$$

where $B_{n, n}$, is a $p X p$ square matrix and $g_{n}$, and $C_{n}$ are column vectors of $p$ elements:

$$
B_{n, n \prime}=\sum_{\alpha} W\left(\theta_{\alpha}\right) p_{n}\left(\theta_{\alpha}\right) p_{n \prime}\left(\theta_{\alpha}\right), \quad C_{n}=\sum_{\alpha} W\left(\theta_{\alpha}\right) p_{n}\left(\theta_{\alpha}\right) G\left(\theta_{\alpha}\right) .
$$

Denote the inverse of $B$ by $B^{-1}$. According to (30)

$$
g=B^{-1} C
$$

and, on substituting the exact expression for $C$ as given by (4), we find

$$
g_{n}=g_{n}^{*}+\sum_{l=p+1}^{\infty} g_{l}^{*} \sum_{n \prime} B_{n, n,}^{-1} \sum_{\alpha} W\left(\theta_{\alpha}\right) p_{l}\left(\theta_{\alpha}\right) p_{n \prime}\left(\theta_{\alpha}\right)
$$

i.e.

$$
g_{n}=g_{n} *+\sum_{l=p+1}^{\infty} D_{n, l} g_{l}^{*}
$$


where $D_{n, l}(n=0$ to $p ; l>p)$ is the solution of the equations

$$
\sum_{n=0}^{n}\left[\Sigma_{\alpha} W\left(\theta_{\alpha}\right) p_{n}\left(\theta_{\alpha}\right) p_{n^{\prime}}\left(\theta_{\alpha}\right)\right] D_{n^{\prime}, l}=\Sigma_{\alpha} W\left(\theta_{\alpha}\right) p_{l}\left(\theta_{\alpha}\right) p_{n}\left(\theta_{\alpha}\right)
$$

It is evident that, if the grid points are equally spaced as the limit $q \rightarrow \infty$ is taken and, if $W(\theta)=\sin \theta$,

$$
\sum_{\alpha} W\left(\theta_{\alpha}\right) p_{n}\left(\theta_{\alpha}\right) p_{n \prime}\left(\theta_{\alpha}\right) \rightarrow(2 s+1) \pi^{-1} \int_{0}^{\pi} p_{n}(\theta) p_{n}(\theta) \sin \theta d \theta=2 \delta_{n}^{n \prime} / \pi
$$

and the coefficients $g_{n} \rightarrow g_{n}{ }^{*}$. However, in practical application, $q$ is generally small, and the deviations between $g_{n}$ and $g_{n}{ }^{*}$ may therefore be significant, as the numerical work below proves.

The claims we have made concerning the superiority of exact fitting techniques to least squares techniques are supported by Tables 1,2 and 3 . In table 1 the coefficients $D_{n, l}(n=0$ to $6, l=7$ to 30 ) are given for the case $p=6$ and $q=9$ (17 equally spaced grid points, excluding poles) and $W(\theta)=\sin \theta$. It is clear that the contribution made by $g_{n}^{*}$ to $g_{1}$ is fairly small. Therefore one is inclined to believe that the inaccuracy of the least square method has little bearing on the question of whether the earth's dipole moment is increasing or not (Bullard, (1953), Macht, (1954); Nagata and Rikitake, (1957)). However, the position is evidently worse for the higher harmonics, particularly $g_{5}$ and $g_{6}$ which are seriously contaminated by $g_{7}$ and $g_{8}$, respectively.

In table 2, the coefficients $\Delta_{n, l}$ ( $n=0$ to 16$),(l=17$ to 30$)$ are given for the case $q=9$ (17 equally spaced grid points, excluding poles). It is clear that the contribution made by $g_{17}^{*}$ to $g_{1}$ is not likely to be large, since $g_{17}^{*}$ may be expected to be small and $\Delta_{1,17}$ is only -0.02271 (see table 2). The fact that the high harmonics (e.g. $g_{16}$ ) are seriously influenced by the higher harmonics (e.g. $g_{18}^{*}$ ) is of no significance since we have already agreed that terms beyond the cut-off point $p(\ll 2 q)$ are of no significance and should be discarded.

In table $3^{+}$, the coefficients $\Delta_{n, l}$ ( $n=0$ to $16, l=19$ to 30 ) are given for the case $q=9$ but now the position of the 17 grid points are given by $p_{17}\left(\theta_{\alpha}\right)=0$. It is clear that this choice of grid points is an enormous improvement even over the choice of equally spaced grid points given in table 2. It is hoped, therefore, that this technique would enable the higher harmonics to be determined reliably.

Clearly, the analysis we have given is applicable to the case of irregularly spaced data points, and again we may be certain that, for extrapolation purposes, the method of exact fitting and discard is preferable to any empirical approach such as least squares.

This paper was read at a meeting of the panel of the World Magnetic Survey held at Washington D.C., U.S.A., on November 13, 1961.

+ In this table, the notation ' $O *$ ' means that the magnitude of the entry concerned lies between $-10^{-7}$ and $+10^{-7}$. 
Table 1. Values of $D_{n, l}(W(\theta)=\sin \theta)$

\begin{tabular}{|c|c|c|c|c|c|c|c|}
\hline$n$ & 7 & 8 & 9 & 10 & 11 & 12 & 13 \\
\hline 0 & 0 & 0.0134 & 0 & -0.01847 & 0 & -0.0102 & 0 \\
\hline 1 & -0.008442 & 0 & -0.008685 & 0 & -0.009008 & 0 & -0.009432 \\
\hline 2 & 0 & -0.05614 & 0 & 0.0243 & 0 & -0.05 & 0 \\
\hline 3 & -0.01988 & 0 & -0.02047 & 0 & -0.02126 & 0 & -0.0223 \\
\hline 4 & 0 & 0.02778 & 0 & -0.07923 & 0 & -0.0157 & 0 \\
\hline 5 & -0.03175 & 0 & -0.03275 & 0 & -0.0341 & 0 & -0.03589 \\
\hline 6 & 0 & -0.1 .074 & 0 & 0.02374 & 0 & -0.0985 & 0 \\
\hline
\end{tabular}

\begin{tabular}{|c|c|c|c|c|c|c|c|}
\hline$n$ & 14 & 15 & 16 & 17 & 18 & 19 & 20 \\
\hline 0 & -0.0166 & 0 & 0.0079 & 0 & -0.0157 & 0 & 0.0059 \\
\hline 1 & 0 & -0.009986 & 0 & -0.01072 & 0 & -0.01169 & 0 \\
\hline 2 & 0.0172 & 0 & -0.047 & 0 & 0.0112 & 0 & -0.047 \\
\hline 3 & 0 & -0.02366 & 0 & -0.02548 & 0 & -0.02794 & 0 \\
\hline 4 & -0.0748 & 0 & 0.0057 & 0 & -0.0753 & 0 & -0.0059 \\
\hline 5 & 0 & -0.03828 & 0 & -0.04151 & 0 & -0.046 & 0 \\
\hline 6 & 0.01 & 0 & -0.097 & 0 & -0.0044 & 0 & -0.1036 \\
\hline
\end{tabular}

\begin{tabular}{|c|c|c|c|c|c|c|c|}
\hline$n$ & 21 & 22 & 23 & 24 & 25 & 26 & 27 \\
\hline 0 & 0 & -0.0158 & 0 & -0.0033 & 0 & -0.0179 & 0 \\
\hline 1 & -0.01303 & 0 & -0.01494 & 0 & -0.01785 & 0 & -0.02273 \\
\hline 2 & 0 & 0.0041 & 0 & -0.0508 & 0 & -0.0071 & 0 \\
\hline 3 & -0.03139 & 0 & -0.03648 & 0 & -0.04457 & 0 & -0.0593 \\
\hline 4 & 0 & -0.0827 & 0 & -0.0259 & 0 & -0.1098 & 0 \\
\hline 5 & -0.05255 & 0 & -0.06283 & 0 & -0.08107 & 0 & -0.1225 \\
\hline 6 & 0 & -0.0267 & 0 & -0.1299 & 0 & -0.0926 & 0 \\
\hline
\end{tabular}

\begin{tabular}{|c|c|c|c|}
\hline & 28 & 29 & 30 \\
\hline 0 & -0.0054 & 0 & -0.011 \\
\hline 1 & 0 & -0.03284 & 0 \\
\hline 2 & -0.0605 & 0 & -0.0732 \\
\hline 3 & 0 & -0.09503 & 0 \\
\hline 4 & -0.1017 & 0 & -0.2725 \\
\hline 5 & 0 & -0.3198 & 0 \\
\hline 6 & -0.3744 & 0 & 0.2494 \\
\hline
\end{tabular}


Table 2. Values of $\Delta_{n, l}$ (equidistant ordinates)

\begin{tabular}{|c|c|c|c|c|c|c|c|}
\hline & 17 & 18 & 19 & 20 & 21 & 22 & 23 \\
\hline 0 & 0 & -0.01472 & 0 & -0.007521 & 0 & -0.006145 & 0 \\
\hline 1 & -0.02271 & 0 & -0.03308 & 0 & -0.02045 & 0 & -0.01814 \\
\hline 2 & 0 & -0.07429 & 0 & -0.03805 & 0 & -0.03127 & 0 \\
\hline 3 & -0.05383 & 0 & -0.07852 & 0 & -0.04889 & 0 & -0.04398 \\
\hline 4 & 0 & -0.1368 & 0 & -0.07051 & 0 & -0.05885 & 0 \\
\hline 5 & -0.08713 & 0 & -0.1275 & 0 & -0.08055 & 0 & -0.07477 \\
\hline 6 & 0 & -0.2053 & 0 & -0.1071 & 0 & -0.09216 & 0 \\
\hline 7 & -0.1244 & 0 & -0.183 & 0 & -0.1189 & 0 & -0.1176 \\
\hline 8 & 0 & -0.2844 & 0 & -0.1516 & 0 & -0.1387 & 0 \\
\hline 9 & -0.1686 & 0 & -0.2505 & 0 & -0.1722 & 0 & -0.1973 \\
\hline 10 & 0 & -0.3821 & 0 & -0.213 & 0 & -0.2247 & 0 \\
\hline 11 & -0.2253 & 0 & -0.3419 & 0 & -0.2684 & 0 & -0.5307 \\
\hline 12 & 0 & -0.516 & 0 & -0.3214 & 0 & -0.5805 & 0 \\
\hline 13 & -0.3071 & 0 & -0.494 & 0 & -0.652 & 0 & 0.3827 \\
\hline 14 & 0 & -0.7386 & 0 & -0.7363 & 0 & 0.3874 & 0 \\
\hline 15 & -0.4559 & 0 & -0.9946 & 0 & 0.3651 & 0 & 0.06845 \\
\hline 16 & 0 & -1.402 & 0 & 0.3309 & 0 & 0.05252 & 0 \\
\hline
\end{tabular}

\begin{tabular}{|c|c|c|c|c|c|c|c|}
\hline$n$ & 24 & 25 & 26 & 27 & 28 & 29 & 30 \\
\hline 0 & -0.005951 & 0 & -0.0065 & 0 & -0.007977 & 0 & -0.01134 \\
\hline 1 & 0 & -0.01869 & 0 & -0.02175 & 0 & -0.02906 & 0 \\
\hline 2 & -0.03062 & 0 & -0.03412 & 0 & -0.04359 & 0 & -0.06857 \\
\hline 3 & 0 & -0.04653 & 0 & -0.05701 & 0 & -0.08614 & 0 \\
\hline 4 & -0.05937 & 0 & -0.07008 & 0 & -0.1025 & 0 & -0.271 \\
\hline 5 & 0 & -0.08413 & 0 & -0.1188 & 0 & -0.3056 & 0 \\
\hline 6 & -0.09905 & 0 & -0.1353 & 0 & -0.3394 & 0 & 0.2596 \\
\hline 7 & 0 & -0.1535 & 0 & -0.3738 & 0 & 0.2825 & 0 \\
\hline 8 & -0.1729 & 0 & -0.4086 & 0 & 0.3044 & 0 & 0.0811 \\
\hline 9 & 0 & -0.4459 & 0 & 0.3245 & 0 & 0.0848 & 0 \\
\hline 10 & -0.485 & 0 & 0.3437 & 0 & 0.08789 & 0 & 0.0421 \\
\hline 11 & 0 & 0.36 & 0 & 0.08925 & 0 & 0.0418 & 0 \\
\hline 12 & 0.375 & 0 & 0.08977 & 0 & 0.04098 & 0 & 0.02317 \\
\hline 13 & 0 & 0.08671 & 0 & 0.03815 & 0 & 0.021 & 0 \\
\hline 14 & 0.08225 & 0 & 0.03465 & 0 & 0.01849 & 0 & 0.01121 \\
\hline 15 & 0 & 0.02691 & 0 & 0.01373 & 0 & 0.0080675 & \\
\hline 16 & 0.0189 & 0 & 0.0091 & 0 & 0.005144 & 0 & 0.0032 \\
\hline
\end{tabular}


Table 3. Values of $\Delta_{n, l}$ (non-equidistant ordinates)

\begin{tabular}{|c|c|c|c|c|c|c|}
\hline$n$ & 19 & 20 & 21 & 22 & 23 & 24 \\
\hline 0 & 0 & $0^{*}$ & 0 & $0 *$ & 0 & $0 *$ \\
\hline 1. & $0 *$ & 0 & $0 *$ & 0 & $0^{*}$ & 0 \\
\hline 2 & 0 & $0 *$ & 0 & $0 *$ & 0 & $0 *$ \\
\hline 3 & $0^{*}$ & 0 & $0 *$ & 0 & $0^{*}$ & 0 \\
\hline 4 & 0 & $0 *$ & 0 & $0^{*}$ & 0 & $0^{*}$ \\
\hline 5 & $0^{*}$ & 0 & $0^{*}$ & 0 & $0 *$ & 0 \\
\hline 6 & 0 & $0^{*}$ & 0 & $0 *$ & 0 & $0 *$ \\
\hline 7 & $0 *$ & 0 & $0 *$ & 0 & $0^{*}$ & 0 \\
\hline 8 & 0 & $0^{*}$ & 0 & $0^{*}$ & 0 & $0^{*}$ \\
\hline 9 & $0 *$ & 0 & $0^{*}$ & 0 & $0 *$ & 0 \\
\hline 10 & 0 & $0 *$ & 0 & $0 *$ & 0 & -0.6564 \\
\hline 11 & $0 *$ & 0 & $0^{*}$ & 0 & -0.7009 & 0 \\
\hline 12 & 0 & $0^{*}$ & 0 & -0.7463 & 0 & -0.002654 \\
\hline 13 & $0 *$ & 0 & -0.793 & 0 & -0.001855 & 0 \\
\hline 14 & 0 & -0.8414 & 0 & -0.001189 & 0 & -0.001665 \\
\hline 15 & -0.8917 & 0 & -0.0006548 & 0 & -0.0009727 & 0 \\
\hline 16 & 0 & -0.0002551 & 0 & -0.000417 & 0 & -0.0005835 \\
\hline$n$ & 25 & 26 & 27 & 28 & 29 & 30 \\
\hline 0 & 0 & $0^{*}$ & 0 & $0 *$ & 0 & $0 *$ \\
\hline 1 & $0^{*}$ & 0 & $0^{*}$ & 0 & $0^{*}$ & 0 \\
\hline 2 & 0 & $0^{*}$ & 0 & $0^{*}$ & 0 & $0 *$ \\
\hline 3 & $0 *$ & 0 & $0 *$ & 0 & $0^{*}$ & 0 \\
\hline 4 & 0 & $0 *$ & 0 & $0 *$ & 0 & -0.3909 \\
\hline 5 & $0^{*}$ & 0 & $0^{*}$ & 0 & -0.4373 & 0 \\
\hline 6 & 0 & $0^{*}$ & 0 & -0.482 & 0 & -0.01177 \\
\hline 7 & $0 *$ & 0 & -0.5259 & 0 & -0.009425 & 0 \\
\hline 8 & 0 & -0.5693 & 0 & -0.00755 & 0 & -0.009781 \\
\hline 9 & -0.6127 & 0 & -0.006004 & 0 & -0.007723 & 0 \\
\hline 10 & 0 & -0.004703 & 0 & -0.006051 & 0 & -0.00773 \\
\hline 11 & -0.003597 & 0 & -0.004661 & 0 & -0.005938 & 0 \\
\hline 12 & 0 & -0.003491 & 0 & -0.004461 & 0 & -0.005647 \\
\hline 13 & -0.0025 & 0 & -0.003225 & 0 & -0.004084 & 0 \\
\hline 14 & 0 & -0.002184 & 0 & -0.00278 & 0 & -0.003499 \\
\hline 15 & -0.001309 & 0 & -0.001684 & 0 & -0.002125 & 0 \\
\hline 16 & 0 & -0.0007642 & 0 & -0.0009708 & 0 & -0.001219 \\
\hline
\end{tabular}




\section{References}

Alldredge, L.R. and Von Voorhis, G.D., Depth of sources of magnetic anomalies. J. Geophys. Res., 66, 3793-3800, 1961.

Bullard, E.C. Is the earth's dipole moment increasing ?, J. Geophys. Res., 58, 277-278, 1953.

Chakrabarty, S.K., The spherical harmonic analysis of the earth's main magnetic field, Indian Journal of Meteorology and Geophysics, 5, 63-68, 1954.

Chapman, S., and J. Bartels. Geomagnetism, Clarendon press, Oxford, 1940.

Courant, R., K. Friedrichs, and H. Lewy, Über die partiellen differenzengleichungen der mathematischen physik, Math. Ann., 100, 32-74, 1928.

Dyson, F., and H. Furner, The Earth's magnetic potential, Mon. Not. R. Astr. Soc. (Geophys. Suppl.), 1, 76-88, 1923.

Fougere, P.F., Spherical Harmonic Analysis 1. A New Method and Its Verification, J. Geophys. Res., 68, 1131-1139, 1963.

Gauss, C.F., Allgemeine theorie des erdmagnetismus, Resultate magn. Verein, 1838.

Jones, H. Spencer, and Melotte, P.J., The harmonic analysis of the earth's magnetic field for epoch, 1942, Mon. Not. R. Astr. Soc. (Geophys. Suppl.), 6, 409-430, 1953.

Kendall, M.G. The Advanced Theory of Statistics, vol. 2, Griffin, 1943.

Lowes, F.J., and Runcorn, S.K., The Analysis of the geomagnetic Secular variation. Phil. Trans. R. Soc Lond., A. 243, 525-546, 1951.

Macht, H.G. On the increase of the earth's dipole moment, J. Geophys. Res., 59, 369-376, 1954.

McNish, A.G., Physical representation of the geomagnetic field, Trans. Am. Geophys. Un. 21. 287-291, 1940.

Nagata, T. and Rikitake, T., Geomagnetic secular variation during the period from 1950-1955, J. Geomag. Geoelec., 9, 42-50, 1957.

Neumman, J. von and Goldstine, H.H., Numerical inverting of matrices of high order. Bull. Am. Math. Soc. 53. 1021-1099, 1947.

Vestine, E.H., Laporte, L., Lange, I. and Scott, W.E., The geomagnetic field: Its description and analysis, Carnegie Institution Publications (Washington), No. 580, 1947. 